\title{
REGULARITY AT INFINITY OF REAL MAPPINGS AND A MORSE-SARD THEOREM
}

\author{
L.R.G. DIAS, M.A.S. RUAS, AND M. TIBĂR
}

\begin{abstract}
We prove a new Morse-Sard type theorem for the asymptotic critical values of semi-algebraic mappings and a new fibration theorem at infinity for $C^{2}$ mappings. We show the equivalence of three different types of regularity conditions which have been used in the literature in order to control the asymptotic behaviour of mappings. The central role of our picture is played by the $t$-regularity and its bridge toward the $\rho$-regularity which implies topological triviality at infinity.
\end{abstract}

\section{INTRODUCTION}

Let $f: \mathbb{K}^{n} \rightarrow \mathbb{K}^{p}$, for $n>p>0$ and $\mathbb{K}=\mathbb{R}$ or $\mathbb{C}$, be a non-constant polynomial mapping. It is well-known that $f$ is a locally trivial topological fibration over the complement of the bifurcation set $B(f)$, also called the set of atypical values. The atypical values may come from the critical values but also from the asymptotic behaviour of the fibres. One can easily see this in the example $f(x, y)=x+x^{2} y$, where the value $0 \in \mathbb{K}$ is not critical but there is no trivial fibration in any neighbourhood of 0 .

A complete characterization of $B(f)$ is available only in the case $n=2$ and $p=1$, see $[$ HaLe] for $\mathbb{K}=\mathbb{C}$ and [TZ] for $\mathbb{K}=\mathbb{R}$. One has therefore imagined various ways to approximate $B(f)$, essentially through the use of regularity conditions at infinity. For $p=$ 1 and $\mathbb{K}=\mathbb{C}$, Broughton worked with a Palais-Smale type condition called tameness $[\mathrm{Br}]$, later extended by Némethi [Ne] and Némethi-Zaharia [NZ] to quasi-tame and M-tame. Parusinski used the Malgrange condition [Pa1], which is a Łojasiewicz type condition at infinity, and versions of it. Siersma and Tibăr worked with the t-regularity (also called $t$-equisingularity) [ST, Ti4], which is a type of non-characteristic condition at infinity, see also [Pa1]. Over $\mathbb{R}$ and still for $p=1$, the $t$-regularity and the $\rho$-regularity were considered in [Ti1, Ti3]. One finds a detailed discussion of the relations between these conditions in [NZ], [Du] for the complex setting and in [Ti3, Ti5] for the real and complex settings.

Let us turn to the case of mappings, i.e. $p>1$. In his study [Ga3] of polynomial mappings $f: \mathbb{C}^{n} \rightarrow \mathbb{C}^{p}$, Gaffney defines a generalized Malgrange condition and proves that this yields a set $A_{G}$ of non-regular values containing $B(f)$. He uses the theory of

Date: November 11, 2018.

2000 Mathematics Subject Classification. 14D06, 51N10, 14P10, 32S20, 32S15, 58K15, 57BN80.

Key words and phrases. Morse-Sard theorem, equisingularity, atypical values, fibration at infinity, regularity at infinity.

LRG Dias acknowledges the Brazilian grants CAPES-Proc. 2929/10-04 and FAPESP-Proc. 2008/10563-4. MAS Ruas acknowledges support from CNPq- Proc. 303774/2008-8 and FAPESP Proc. 08/54222-6. M Tibăr acknowledges support from the French grant ANR-08-JCJC-0118-01. 
integral closure of modules to relate his condition to a non-characteristic condition like Parusinski's [Pa1].

In [KOS], Kurdyka, Orro and Simon considered $C^{2}$ semi-algebraic maps $f: \mathbb{K}^{n} \rightarrow \mathbb{K}^{p}$ and a metric type regularity condition which had been introduced by Rabier [Ra]. They define a set of asymptotically critical values $K_{\infty}(f)$ and show the inclusion $A_{K O S} \supset B(f)$, where $A_{K O S}:=f(\operatorname{Sing} f) \cup K_{\infty}(f)$. Remarkably, they prove that $K_{\infty}(f)$ is a semi-algebraic set of dimension $\leq p-1$. Their condition, which we shall call here KOS-regularity, is a different extension of the Malgrange condition. It was established by Jelonek [Je1, Je2] that these two generalizations of the Malgrange condition are actually equivalent, see Remark 4.5.

In each of the above cases, showing that some set $A$ of non-regular values is semialgebraic (or algebraic, in the complex case) of dimension $\leq p-1$ and contains $B(f)$, means to prove an asymptotic Morse-Sard type theorem together with a fibration theorem for the non-proper mapping $f$.

In our paper, the central object is the t-regularity in the setting of semi-algebraic $C^{2}$ mappings $f: \mathbb{R}^{n} \rightarrow \mathbb{R}^{p}$, which is a geometric grounded condition. Following Gaffney, the interpretation in terms of integral closure of modules (Proposition 4.3) allows one to prove that the $t$-regularity is equivalent to the Malgrange-Gaffney condition reformulated over $\mathbb{R}$ (Theorem 4.4). Due to the technique, this holds within the class of "fair" maps [Ga3, p. 159 and Prop. 2], see also §4.2.

Our Theorem 3.1 tells that the $t$-regularity is equivalent to the $K O S$-regularity, providing a bridge between conditions of very different flavor. This new result together with Jelonek's above mentioned theorem establishes a "triangle" of equivalences in Figure 1. It also shows that the equivalence of the $t$-regularity with the Malgrange-Gaffney condition holds under general conditions, i.e. without the "fair" assumption and without using at all the integral closure interpretation, see Example 5.10. This is also a far reaching extension of the equivalence proved for $p=1$ in [ST, Pa2].

We pursue by showing that $t$-regularity implies $\rho_{E}$-regularity, a Milnor type condition of transversality of $f$ to the Euclidean distance function $\rho_{E}$, extending a result proved for $p=1$ in [Ti3]. The $\rho$-regularity enables one to define the set of asymptotic non $\rho$-regular values $S(f) \subset \mathbb{R}^{p}$, and the set $A_{\rho}:=f(\operatorname{Sing} f) \cup S(f)$.

Then Theorem 5.7 tells that the subset $S(f)$ is closed semi-algebraic and of dimension $\leq p-1$. Moreover, Theorem 5.7(c) shows that there is a locally trivial fibration induced by $f$ on the complement $\mathbb{R}^{p} \backslash A_{\rho_{E}}$, under the more general setting of a $C^{2}$-mapping $f: X \rightarrow \mathbb{R}^{p}$ on a submanifold $X \subset \mathbb{R}^{n}$. Our fibration result is based on a new result, Proposition 6.4, which is a fibration theorem "at infinity", i.e. holding in the complement of a sufficiently large ball. We show in Corollary 5.8 the inclusions $A_{\rho_{E}} \subset A_{K O S}$, and more particularly $S(f) \subset K_{\infty}(f)$, which inclusions may be strict, cf Example 5.9. Therefore Theorem 5.7 represents an asymptotic Morse-Sard type theorem which refines the one by Kurdyka, Orro and Simon [KOS]. In particular, the key result $\operatorname{dim} K_{\infty}(f) \leq p-1$ of [KOS] is superseded by $\operatorname{dim} S(f) \leq p-1$ (cf Theorem 5.7(b)). Our proof is of a completely different flavor and is based on the existence of partial Thom stratifications at infinity, cf Definition 6.2 , and on the link between $t$-regularity and $\rho$-regularity. 
Let us also point out that our definitions of regularity conditions as well as our statements involving them have a local-at-infinity counterpart, i.e. formulated at some fixed point $p_{0}$ on the boundary at infinity of the graph of $f$ in $\mathbb{P}^{n} \times \mathbb{R}^{p}$.

Figure 1 presents our results in a condensed manner.

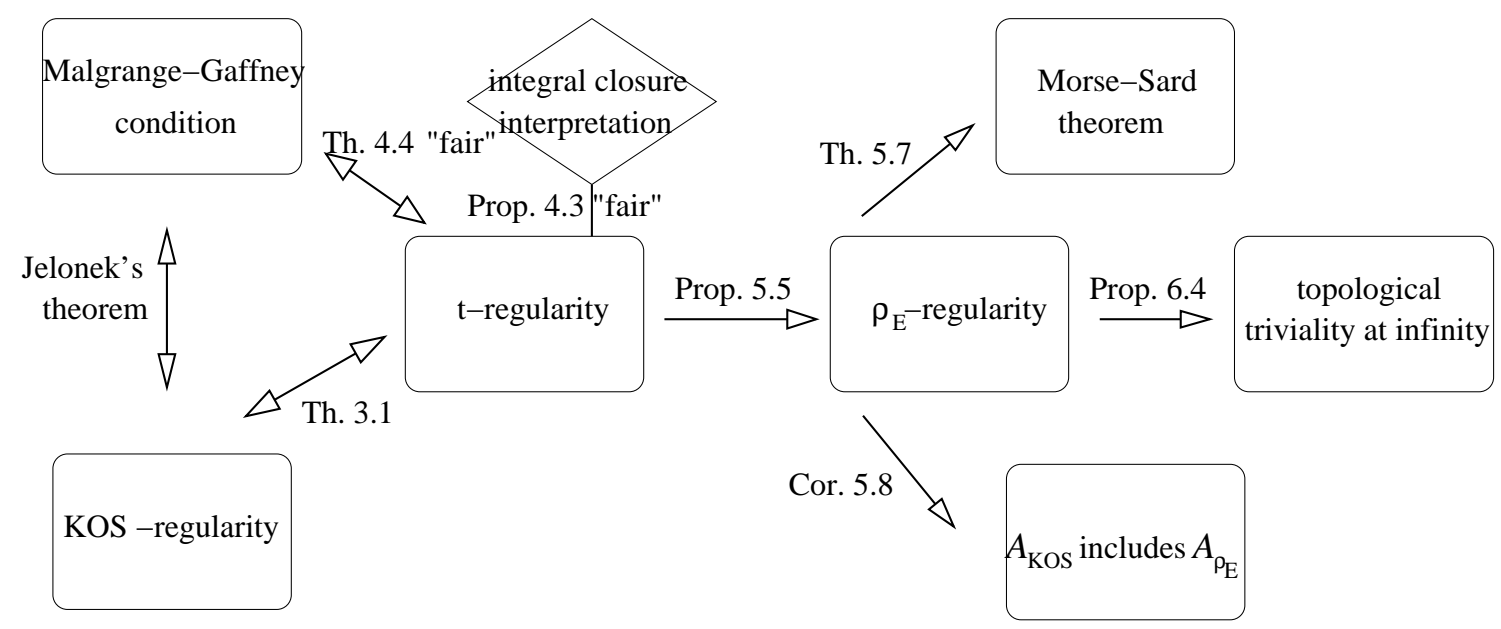

Figure 1. Synopsis

\section{ASYMPTOTIC REGULARITY CONDITIONS FOR MAPPINGS}

We introduce the main definitions leading to the notion of $t$-regularity, then define the Malgrange-Gaffney regularity and the KOS-regularity.

2.1. Conormal spaces. Let $X \subset \mathbb{R}^{m}$ be a real semi-algebraic subset. We denote by $X_{\text {reg }}$ the set of regular points of $X$ and by $X_{\text {sing }}$ the set of singular points of $X$. We assume that $X$ contains at least a regular point.

Definition 2.1. Let

$$
C(X):=\text { closure }\left\{(x, H) \in X_{\text {reg }} \times \check{\mathbb{P}}^{m-1} \mid T_{x} X_{\text {reg }} \subset H\right\} \subset \bar{X} \times \check{\mathbb{P}}^{m-1}
$$

be the conormal modification of $X$. Let $\pi: C(X) \rightarrow \bar{X}$ denote the projection.

Definition 2.2. Let $g: X \rightarrow \mathbb{R}$ be an analytic function defined in some neighbourhood of $X$ in $\mathbb{R}^{m}$. Let $X_{0}$ denote the subset of $X_{\text {reg }}$ where $g$ is a submersion. The relative conormal space of $g$ is defined as follows:

$$
C_{g}(X):=\operatorname{closure}\left\{(x, H) \in X_{0} \times \check{\mathbb{P}}^{m-1} \mid T_{x}\left(g^{-1}(g(x))\right) \subset H\right\} \subset \bar{X} \times \check{\mathbb{P}}^{m-1},
$$

together with the projection $\pi: C_{g}(X) \rightarrow \bar{X}, \pi(x, H)=x$.

For some $y \in \bar{X}$ such that $g(y)=0$, let $C_{g, y}(X):=\pi^{-1}(y)$. The following result shows that $C_{g, y}(X)$ depends on the germ of $g$ at $y$ only up to multiplication by some invertible analytic function germ $\gamma$. It was stated for analytic $X$ but holds for semi-algebraic. 
Lemma 2.3. [Ti5, Lemma 1.2.7]

Let $\gamma:\left(\mathbb{R}^{m}, y\right) \rightarrow \mathbb{R}$ be an analytic function such that $\gamma(y) \neq 0$. Then $C_{\gamma g, y}(X)=C_{g, y}(X)$.

2.2. Characteristic covectors and t-regularity. Let $X \subset \mathbb{R}^{n}$ be semi-algebraic and let $f: X \rightarrow \mathbb{R}^{p}$ be a semi-algebraic $C^{1}$ mapping with $n>p$. We use coordinates $\left(x_{1}, \ldots, x_{n}\right)$ for the space $\mathbb{R}^{n}$ and coordinates $\left[x_{0}: x_{1}: \ldots: x_{n}\right]$ for the projective space $\mathbb{P}^{n}$. We denote by $H^{\infty}=\left\{\left[x_{0}: x_{1}: \ldots: x_{n}\right] \in \mathbb{P}^{n} \mid x_{0}=0\right\}$ the hyperplane at infinity.

Let $\mathbb{X}:=\overline{\text { graph } f}$ be the closure of the graph of $f$ in $\mathbb{P}^{n} \times \mathbb{R}^{p}$ and let $\mathbb{X}^{\infty}:=\mathbb{X} \cap\left(H^{\infty} \times \mathbb{R}^{p}\right)$. One has the semi-algebraic isomorphism graph $f \simeq X$.

We consider the affine charts $U_{j} \times \mathbb{R}^{p}$ of $\mathbb{P}^{n} \times \mathbb{R}^{p}$, where $U_{j}=\left\{x_{j} \neq 0\right\}, j=0,1, \ldots, n$. Identifying the chart $U_{0}$ with the affine space $\mathbb{R}^{n}$, we have $\mathbb{X} \cap\left(U_{0} \times \mathbb{R}^{p}\right)=\mathbb{X} \backslash \mathbb{X}^{\infty}=$ graph $f$ and $\mathbb{X}^{\infty}$ is covered by the charts $U_{1} \times \mathbb{R}^{p}, \ldots, U_{n} \times \mathbb{R}^{p}$.

If $g$ denotes the projection to the variable $x_{0}$ in some affine chart $U_{j} \times \mathbb{R}^{p}$, then the relative conormal $C_{g}\left(\mathbb{X} \backslash \mathbb{X}^{\infty} \cap U_{j} \times \mathbb{R}^{p}\right) \subset \mathbb{X} \times \check{\mathbb{P}}^{n+p-1}$ is well defined, with the projection $\pi(y, H)=y$. Let us then consider the space $\pi^{-1}\left(\mathbb{X}^{\infty}\right)$ which is well defined for every chart $U_{j} \times \mathbb{R}^{p}$ as a subset of $C_{g}\left(\mathbb{X} \backslash \mathbb{X}^{\infty} \cap U_{j} \times \mathbb{R}^{p}\right)$. By Lemma 2.3, the definitions coincide at the intersections of the charts. We therefore have:

Definition 2.4. We call space of characteristic covectors at infinity the well-defined set $\mathcal{C}^{\infty}:=\pi^{-1}\left(\mathbb{X}^{\infty}\right)$. For some $p_{0} \in \mathbb{X}^{\infty}$, we denote $\mathcal{C}_{p_{0}}^{\infty}:=\pi^{-1}\left(p_{0}\right)$.

Let $\tau: \mathbb{P}^{n} \times \mathbb{R}^{p} \rightarrow \mathbb{R}^{p}$ denote the second projection. One defines the relative conormal space $C_{\tau}\left(\mathbb{P}^{n} \times \mathbb{R}^{p}\right)$ like in Definition 2.2 where the function $g$ is replaced by the application $\tau$.

Definition 2.5. We say that $f$ is t-regular at $p_{0} \in \mathbb{X}^{\infty}$ if $C_{\tau}\left(\mathbb{P}^{n} \times \mathbb{R}^{p}\right) \cap \mathcal{C}_{p_{0}}^{\infty}=\emptyset$.

2.3. Interpretation of the $t$-regularity in local charts. Let $f=\left(f_{1}, \ldots, f_{p}\right): \mathbb{R}^{n} \rightarrow$ $\mathbb{R}^{p}$ be a semi-algebraic $C^{1}$ mapping and let $p_{0} \in \mathbb{X}^{\infty}$. Up to some linear change of coordinate one may assume that $p_{0} \in \mathbb{X}^{\infty} \cap\left(U_{n} \times \mathbb{R}^{p}\right)$. In the intersection of charts $\left(U_{0} \cap U_{n}\right) \times \mathbb{R}^{p}$, one considers the change of coordinates $x_{1}=y_{1} / y_{0}, \ldots, x_{n-1}=y_{n-1} / y_{0}$, $x_{n}=1 / y_{0}$, where $\left(x_{1}, \ldots, x_{n}\right)$ are the coordinates in $U_{0}$ and $\left(y_{0}, \ldots, y_{n-1}\right)$ are those in $U_{n}$. Then for $i=1, \ldots, p$, we define:

$$
F_{i}(y, t)=F_{i}\left(y_{0}, y_{1}, \ldots, y_{n-1}, t_{1}, \ldots, t_{p}\right):=f_{i}\left(\frac{y_{1}}{y_{0}}, \ldots, \frac{y_{n-1}}{y_{0}}, \frac{1}{y_{0}}\right)-t_{i}
$$

and $F(y, t):=\left(F_{1}(y, t), \ldots, F_{p}(y, t)\right)$. Then $\mathbb{X} \cap\left(\left(U_{0} \cap U_{n}\right) \times \mathbb{R}^{p}\right)=\bigcap_{i=1}^{p}\left\{F_{i}(y, t)=0\right\}$.

Let $\overrightarrow{n_{0}}=(1,0, \ldots, 0) \in \mathbb{R}^{n} \times \mathbb{R}^{p}$ denote a normal vector to the hypersurface $\left\{y_{0}=\right.$ constant $\}$ and, for $i=1, \ldots, p$, let us consider a normal vector to $\left\{F_{i}=0\right\}$ at $(y, t) \in$ $\mathbb{X} \cap\left(\left(U_{0} \cap U_{n}\right) \times \mathbb{R}^{p}\right)$, as follows:

$$
\overrightarrow{n_{i}}(y, t)=\nabla F_{i}(y, t)=\left(\nabla_{n} F_{i}(y, t), \nabla_{p} F_{i}(y, t)\right)
$$

where

$$
\nabla_{n} F_{i}(y, t):=\left(\frac{\partial F_{i}}{\partial y_{0}}(y, t), \ldots, \frac{\partial F_{i}}{\partial y_{n-1}}(y, t)\right), \quad \nabla_{p} F_{i}(y, t):=\left(\frac{\partial F_{i}}{\partial t_{1}}(y, t), \ldots, \frac{\partial F_{i}}{\partial t_{p}}(y, t)\right) .
$$


By Definition 2.5, $f$ is not $t$-regular at $p_{0} \in \mathbb{X}^{\infty}$ if and only if there exists a sequence $\left\{\left(y_{k}, t_{k}\right)\right\}_{k \in \mathbb{N}} \subset \mathbb{X} \cap\left(\left(U_{0} \cap U_{n}\right) \times \mathbb{R}^{p}\right)$ such that $\left(y_{k}, t_{k}\right) \rightarrow p_{0}$ and the tangent hyperplanes to the fibres of $g_{\mid \mathbb{X}}$ at $\left(y_{k}, t_{k}\right)$ tend to a hyperplane $H$ such that its normal line has a direction of the form $\left[0: \cdots: 0: b_{1}: \cdots: b_{p}\right]$ in $\mathbb{P}^{n+p-1}$. More explicitly, there exists a

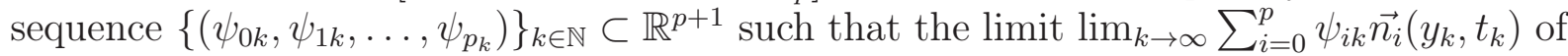
the linear combination of normal vectors $\overrightarrow{n_{i}}$ has the direction $\vec{n}_{H}=\left[0: 0: \ldots: 0: b_{1}\right.$ : $\left.\ldots: b_{p}\right] \in \mathbb{P}^{n+p-1}$.

2.4. KOS-regularity. Kurdyka, Orro and Simon defined in [KOS] a regularity condition at infinity for $C^{1}$ mappings $f: \mathbb{R}^{n} \rightarrow \mathbb{R}^{p}$ which contains the Rabier function $\nu$, cf [Ra], and compared it to the Kuo distance function [Ku]. Jelonek [Je1, Je2] compared it to the generalized Gaffney function, see Remark 4.5.

Let $f: \mathbb{R}^{n} \rightarrow \mathbb{R}^{p}$ be a nonconstant semi-algebraic $C^{1}$ mapping, where $n>p$.

Definition 2.6. [KOS] The set of asymptotic critical values of $f$ is defined as:

$$
\begin{aligned}
K_{\infty}(f):= & \left\{t \in \mathbb{R}^{p} \mid \exists\left\{\mathrm{x}_{j}\right\}_{j \in \mathbb{N}} \subset \mathbb{R}^{n}, \lim _{j \rightarrow \infty}\left\|\mathrm{x}_{j}\right\|=\infty,\right. \\
& \left.\lim _{j \rightarrow \infty} f\left(\mathrm{x}_{j}\right)=t \text { and } \lim _{j \rightarrow \infty}\left\|\mathrm{x}_{j}\right\| \nu\left(\mathrm{D} f\left(\mathrm{x}_{j}\right)\right)=0\right\}
\end{aligned}
$$

where $\nu(A):=\inf _{\|\varphi\|=1}\left\|A^{*}(\varphi)\right\|$, for $A \in \mathcal{L}\left(\mathbb{R}^{n}, \mathbb{R}^{p}\right)$.

We shall reformulate this condition in a localized version, at some point at infinity $p_{0} \in \mathbb{X}^{\infty}$.

Definition 2.7. Let graph $f \subset \mathbb{X} \subset \mathbb{P}^{n} \times \mathbb{R}^{p}$ be a fixed embedding. We say that $p_{0} \in \mathbb{X}^{\infty}$ is an asymptotical critical point of $f$ if and only if there exists $\left\{\mathrm{x}_{j}\right\}_{j \in \mathbb{N}} \subset \mathbb{R}^{n} \simeq$ graph $f$ such that $\lim _{j \rightarrow \infty}\left(\mathrm{x}_{j}, f\left(\mathrm{x}_{j}\right)\right)=p_{0}$ and that $\tau\left(p_{0}\right) \in K_{\infty}(f)$.

We say that $t_{0} \in \mathbb{R}^{p}$ is a $K O S$-regular value of $f$ if $t_{0} \notin K_{\infty}(f)$. We say that $p_{0} \in \mathbb{X}^{\infty}$ is a KOS-regular point whenever $p_{0}$ is not an asymptotical critical point of $f$.

Let us remark that the notion of $K O S$-regular point depends on the embedding of $\mathbb{R}^{n}$, whereas the $K O S$-regular values do not.

2.5. Malgrange-Gaffney condition. Following [Ga3], let $f: \mathbb{R}^{n} \rightarrow \mathbb{R}^{p}$ be a semialgebraic $C^{1}$ mapping and let $I$ be multi-index with $I=\left(i_{1}<i_{2}<\ldots<i_{p}\right)$. One denotes by $M_{I}(f)$ the maximal minor of the Jacobian matrix of $f$ formed from the columns indexed by $I$ and by $M_{J}(j, f)$ the minor of the Jacobian matrix of size $(p-1) \times(p-1)$ using the columns indexed by $J$, and all the rows of the Jacobian matrix except for the $j$-th row. If $p=1$, then, by convention, $M_{J}(j, f)=1$.

Definition 2.8. Let $\left\{\mathrm{x}_{k}\right\}_{k \in \mathbb{N}}$ be a sequence of points in $\mathbb{R}^{n}$ and let us consider the following properties:

$\left(L_{1}\right)\left\|\mathrm{x}_{k}\right\| \rightarrow \infty$ and $f\left(\mathrm{x}_{k}\right) \rightarrow t_{0}$, as $k \rightarrow \infty$.

$\left(L_{2}\right)\left(\mathrm{x}_{k}, f\left(\mathrm{x}_{k}\right)\right) \rightarrow p_{0} \in \mathbb{X}^{\infty}$, as $k \rightarrow \infty$.

After [Ga3], we say that the fibre $f^{-1}\left(t_{0}\right)$ verifies the Malgrange-Gaffney condition if there is $\delta>0$ such that, for any sequences of points $\left\{\mathrm{x}_{k}\right\}_{k \in \mathbb{N}}$ with property $\left(L_{1}\right)$ one has

$$
\left\|\mathrm{x}_{k}\right\| \frac{\left(\sum_{I}\left\|M_{I}(f)\left(\mathrm{x}_{k}\right)\right\|^{2}\right)^{\frac{1}{2}}}{\left(\sum_{J, j}\left\|M_{J}(j, f)\left(\mathrm{x}_{k}\right)\right\|^{2}\right)^{\frac{1}{2}}} \geq \delta .
$$


Localizing this condition, we shall say that $f$ verifies the Malgrange-Gaffney condition at $p_{0} \in \mathbb{X}^{\infty}$ if there is $\delta>0$ such that the above inequality holds for any sequences of points with property $\left(L_{2}\right)$.

It follows that $f^{-1}\left(t_{0}\right)$ verifies the Malgrange-Gaffney condition if and only if $f$ verifies the generalized Malgrange-Gaffney condition at any point $p_{0} \in \tau^{-1}\left(t_{0}\right) \cap \mathbb{X}^{\infty}$.

\section{EQUivalenCE of REGULARITY CONDitions}

With the above definitions and notations, we have:

Theorem 3.1. Let $f: \mathbb{R}^{n} \rightarrow \mathbb{R}^{p}$ be a non-trivial semi-algebraic $C^{1}$ mapping, $n>p>0$. Let $p_{0} \in \mathbb{X}^{\infty}$. Then the following are equivalent:

(a) $f$ is t-regular at $p_{0}$.

(b) $f$ is KOS-regular at $p_{0}$.

(c) $f$ verifies the Malgrange-Gaffney condition at $p_{0}$.

Proof. We shall prove $(\mathrm{a}) \Leftrightarrow(\mathrm{b})$.

One may assume (eventually after some linear change of coordinates) that $p_{0} \in \mathbb{X}^{\infty} \cap$ $\left(U_{n} \times \mathbb{R}^{p}\right)$ and that we have $\left|x_{n}\right| \geq\left|x_{i}\right|, i=1, \ldots, n-1$, for $\mathrm{x} \in \mathbb{R}^{n}$ in some neighbourhood of $p_{0}$.

$(\mathbf{a}) \Rightarrow(\mathbf{b})$. By definition, if $p_{0}$ is an asymptotical critical point of $f$ and therefore $t_{0}:=\tau\left(p_{0}\right)$ is an asymptotical critical value, then there exist sequences $\left\{\mathrm{x}_{k}:=\right.$ $\left.\left(x_{1 k}, \ldots, x_{n k}\right)\right\}_{k \in \mathbb{N}} \subset \mathbb{R}^{n}$ and $\left\{\psi_{k}=\left(\psi_{1 k}, \ldots, \psi_{p k}\right)\right\}_{k \in \mathbb{N}} \subset \mathbb{R}^{p}$ with $\left\|\psi_{k}\right\|=1$ and $\psi_{k} \rightarrow \psi$, such that $\left(\mathrm{x}_{k}, f\left(\mathrm{x}_{k}\right)\right) \rightarrow p_{0}$ and that:

$$
\left\|\mathrm{x}_{k}\right\|\left\|\left(\sum_{i=1}^{p} \psi_{i k} \frac{\partial f_{i}}{\partial x_{1}}\left(\mathrm{x}_{k}\right), \ldots, \sum_{i=1}^{p} \psi_{i k} \frac{\partial f_{i}}{\partial x_{n}}\left(\mathrm{x}_{k}\right)\right)\right\| \rightarrow 0
$$

Since for large enough $k$ we have $\left|x_{n k}\right| \geq\left|x_{i k}\right|, i=1, \ldots, n-1$, one may replace in (2) $\left\|\mathrm{x}_{k}\right\|$ by $\left|x_{n k}\right|$, then multiply the sums by $x_{n k}$.

In the notations of $\S 2.3$, by changing coordinates within $U_{0} \cap U_{n}$, one has $y_{0}=1 / x_{n}, y_{i}=$ $x_{i} / x_{n}$ and the relations:

$$
\left\{\begin{array}{lll}
\frac{\partial F_{j}}{\partial y_{i}}(y, t)=x_{n} \frac{\partial f_{j}}{\partial x_{i}}(x), & & 1 \leq i \leq n-1,1 \leq j \leq p, \\
\frac{\partial F_{j}}{\partial t_{l}}(y, t)=-\delta_{l, j}, & & 1 \leq j, l \leq p, \\
\frac{\partial F_{j}}{\partial y_{0}}(y, t)=-x_{n}\left(x_{1} \frac{\partial f_{j}}{\partial x_{1}}(x)+\ldots+x_{n} \frac{\partial f_{j}}{\partial x_{n}}(x)\right), & & 1 \leq j \leq p .
\end{array}\right.
$$

Then the condition (2) implies:

$$
\left\|\left(\sum_{i=1}^{p} \psi_{i k} \frac{\partial F_{i}}{\partial y_{1}}\left(\mathrm{y}_{k}, \mathrm{t}_{k}\right), \ldots, \sum_{i=1}^{p} \psi_{i k} \frac{\partial F_{i}}{\partial y_{n-1}}\left(\mathrm{y}_{k}, \mathrm{t}_{k}\right)\right)\right\| \rightarrow 0 .
$$

The vector

$$
\vec{n}_{H_{k}}:=\left(0, \sum_{i=1}^{p} \psi_{i k} \frac{\partial F_{i}}{\partial y_{1}}\left(\mathrm{y}_{k}, \mathrm{t}_{k}\right), \ldots, \sum_{i=1}^{p} \psi_{i k} \frac{\partial F_{i}}{\partial y_{n-1}}\left(\mathrm{y}_{k}, \mathrm{t}_{k}\right),-\psi_{1 k}, \ldots,-\psi_{p k}\right)
$$


is a linear combination of the normal vectors $\vec{n}_{i}$ defined in $\S 2.3$ with coefficients $\psi_{i k}$, thus the hyperplanes $H_{k}$ are tangent to the levels of the function $g_{\mid \mathbb{X}}$. We have

$$
\vec{n}_{H_{k}} \rightarrow \vec{n}=\left[0: 0: \ldots: 0: \psi_{1}: \ldots: \psi_{p}\right]
$$

which shows that the limit tangent hyperplane $H=\lim _{k \rightarrow \infty} H_{k}$, to which $\vec{n}$ is normal, belongs to $\mathcal{C}_{p_{0}}^{\infty}$. This implies that $f$ is not $t$-regular at $p_{0}$.

(b) $\Rightarrow$ (a). Reciprocally, let $p_{0} \in \mathbb{X}^{\infty}$ be not $t$-regular. Then there exist some sequence of points $\left\{\left(\mathrm{y}_{k}, \mathrm{t}_{k}\right)\right\}_{k \in \mathbb{N}} \subset \mathbb{X} \cap\left(\left(U_{0} \cap U_{n}\right) \times \mathbb{R}^{p}\right)$ tending to $p_{0}$, and a sequence of hyperplanes $H_{k}$ tangent to the levels of $g$ at $\left(\mathrm{y}_{k}, \mathrm{t}_{k}\right)$, such that $H_{k} \rightarrow H \in \mathcal{C}_{p_{0}}^{\infty}$. This means that there exist sequences $\left\{\tilde{\psi}_{k}=\left(\tilde{\psi}_{1 k}, \ldots, \tilde{\psi}_{p k}\right)\right\}_{k \in \mathbb{N}} \subset \mathbb{R}^{p}$ and $\left\{\lambda_{k}\right\}_{k \in \mathbb{N}} \subset \mathbb{R}$ such that $\vec{n}_{H_{k}}=$ $\lambda_{k} \vec{n}_{0}\left(\mathrm{y}_{k}, \mathrm{t}_{k}\right)+\sum_{i} \tilde{\psi}_{i k} \vec{n}_{i}\left(\mathrm{y}_{k}, \mathrm{t}_{k}\right)$ and that $\lim _{k \rightarrow \infty} \vec{n}_{H_{k}}=\left[0: 0: \ldots: 0: \tilde{\psi}_{1}: \ldots: \tilde{\psi}_{p}\right]$, where $\left(\tilde{\psi}_{1}, \ldots, \tilde{\psi}_{p}\right) \neq(0, \ldots, 0)$. By assumption we have that $\vec{n}_{H_{k}}$ is the vector:

$$
\left(\lambda_{k}+\sum_{i=1}^{p} \tilde{\psi}_{i k} \frac{\partial F_{i}}{\partial y_{0}}\left(\mathrm{y}_{k}, \mathrm{t}_{k}\right), \sum_{i=1}^{p} \tilde{\psi}_{i k} \frac{\partial F_{i}}{\partial y_{1}}\left(\mathrm{y}_{k}, \mathrm{t}_{k}\right), \ldots, \sum_{i=1}^{p} \tilde{\psi}_{i k} \frac{\partial F_{i}}{\partial y_{n-1}}\left(\mathrm{y}_{k}, \mathrm{t}_{k}\right),-\tilde{\psi}_{1 k}, \ldots,-\tilde{\psi}_{p k}\right) .
$$

We may actually take $\lambda_{k}:=-\sum_{i=1}^{p} \tilde{\psi}_{i k} \frac{\partial F_{i}}{\partial y_{0}}\left(\mathrm{y}_{k}, \mathrm{t}_{k}\right)$ and we have, after dividing out by $\mu_{k}:=\left\|\left(\tilde{\psi}_{1 k}, \ldots, \tilde{\psi}_{p k}\right)\right\|$, that $\lim _{k \rightarrow \infty} \vec{n}_{H_{k}}=\left(0,0, \ldots, 0, \psi_{1}, \ldots, \psi_{p}\right)$ where $\psi_{i k}:=\frac{\tilde{\psi}_{i k}}{\mu_{k}}$ and $\left\|\left(\psi_{1 k}, \ldots, \psi_{p k}\right)\right\|=1$. This implies that:

$$
\lim _{k \rightarrow \infty} \sum_{i=1}^{p} \psi_{i k} \frac{\partial F_{i}}{\partial y_{j}}\left(\mathrm{y}_{k}, \mathrm{t}_{k}\right)=0
$$

for any $1 \leq j \leq n-1$.

By using (3), this is equivalent to:

$$
\lim _{k \rightarrow \infty} x_{n k} \sum_{i=1}^{p} \psi_{i k} \frac{\partial f_{i}}{\partial x_{j}}\left(\mathrm{x}_{k}\right)=0
$$

and one has $\left|x_{n k}\right| \geq \frac{1}{\sqrt{n}}\left\|\mathrm{x}_{k}\right\|$ for large enough $k$. Therefore, in order to get the limit (2) it remains to prove that (6) is true for $j=n$. The rest of our argument is devoted to this proof.

From the relations (3) we get $x_{n} \frac{\partial f_{i}}{\partial x_{n}}(x)=-\sum_{j=0}^{n-1} y_{j} \frac{\partial F_{i}}{\partial y_{j}}(y, t)$ and therefore:

$$
\sum_{i=1}^{p} \psi_{i k} x_{n k} \frac{\partial f_{i}}{\partial x_{n}}\left(\mathrm{x}_{k}\right)=-\sum_{j=1}^{n-1} \sum_{i=1}^{p} y_{j k} \psi_{i k} \frac{\partial F_{i}}{\partial y_{j}}\left(\mathrm{y}_{k}, \mathrm{t}_{k}\right)-\sum_{i=1}^{p} \psi_{i k} y_{0 k} \frac{\partial F_{i}}{\partial y_{0}}\left(\mathrm{y}_{k}, \mathrm{t}_{k}\right) .
$$

We show that both terms of the right hand side tend to zero. First we have:

$$
\left|\sum_{j=1}^{n-1} \sum_{i=1}^{p} y_{j k} \psi_{i k} \frac{\partial F_{i}}{\partial y_{j}}\left(\mathrm{y}_{k}, \mathrm{t}_{k}\right)\right| \leq\left\|\frac{\mathrm{x}_{k}}{x_{n k}}\right\|\left\|\left(\sum_{i=1}^{p} \psi_{i k} \frac{\partial F_{i}}{\partial y_{1}}\left(\mathrm{y}_{k}, \mathrm{t}_{k}\right), \ldots, \sum_{i=1}^{p} \psi_{i k} \frac{\partial F_{i}}{\partial y_{n-1}}\left(\mathrm{y}_{k}, \mathrm{t}_{k}\right)\right)\right\| .
$$

Since by hypothesis we have $\left|y_{j k}\right|=\left|\frac{x_{j k}}{x_{n k}}\right| \leq 1$ for large enough $k$, we get from (6) that the right hand side tends to 0 as $k \rightarrow \infty$. 
Let us assume that the following inequality holds for large enough $k \gg 1$, the proof of which will be given below:

$$
\left\|\sum_{i=1}^{p} \psi_{i k} y_{0 k} \frac{\partial F_{i}}{\partial y_{0}}\right\| \ll\left\|\left(\sum_{i=1}^{p} \psi_{i k} \frac{\partial F_{i}}{\partial y_{1}}, \ldots, \sum_{i=1}^{p} \psi_{i k} \frac{\partial F_{i}}{\partial y_{n-1}}, \sum_{i=1}^{p} \psi_{i k} \frac{\partial F_{i}}{\partial t_{1}}, \ldots, \sum_{i=1}^{p} \psi_{i k} \frac{\partial F_{i}}{\partial t_{p}}\right)\right\| .
$$

Then, by using (5), (7) and the equality $\sum_{i=1}^{p} \psi_{i k} \frac{\partial F_{i}}{\partial t_{j}}=-\psi_{j k}$ for any $1 \leq j \leq p$ (implied by (3)), we get:

$$
\left\|\sum_{i=1}^{p} \psi_{i k} y_{0 k} \frac{\partial F_{i}}{\partial y_{0}}\right\| \ll\left\|\psi_{k}\right\|=1
$$

which shows that $\lim _{k \rightarrow \infty} \sum_{i=1}^{p}\left\|\psi_{i k} y_{0 k} \frac{\partial F_{i}}{\partial y_{0}}\left(\mathrm{y}_{k}, \mathrm{t}_{k}\right)\right\|=0$. This completes our proof of the relation (2) showing that $p_{0}$ is not $K O S$-regular.

Let us now give the proof of (7). If this were not true, there exists $\delta>0$ such that for $k \gg 1$ one has:

$$
\frac{\left\|\sum_{i=1}^{p} \psi_{i k} y_{0 k} \frac{\partial F_{i}}{\partial y_{0}}\left(\mathrm{y}_{k}, \mathrm{t}_{k}\right)\right\|}{\left\|\left(\sum_{i=1}^{p} \psi_{i k} \frac{\partial F_{i}}{\partial y_{1}}, \ldots, \sum_{i=1}^{p} \psi_{i k} \frac{\partial F_{i}}{\partial y_{n-1}}, \sum_{i=1}^{p} \psi_{i k} \frac{\partial F_{i}}{\partial t_{1}}, \ldots, \sum_{i=1}^{p} \psi_{i k} \frac{\partial F_{i}}{\partial t_{p}}\right)\left(\mathrm{y}_{k}, \mathrm{t}_{k}\right)\right\|}>\delta
$$

Then the set $\mathcal{W}=\left\{((y, t), \psi) \in\left(\left(U_{n} \cap U_{0}\right) \times \mathbb{R}^{p} \times \mathbb{R}^{p}\right) \cap\left(\mathbb{X} \times S_{1}^{p-1}\right) \mid(8)\right.$ holds for $\left.((y, t), \psi)\right\}$ is a semi-algebraic set. We have $\left(\left(\mathrm{y}_{k}, \mathrm{t}_{k}\right), \psi_{k}\right) \in \mathcal{W}$ for $k \gg 1$, thus $\left(p_{0}, \psi\right) \in$ $\overline{\mathcal{W}}$. Then, by the curve selection lemma [Mi, p. 25] there exists an analytic curve $\lambda=(\phi, \psi):\left[0, \varepsilon\left[\rightarrow \overline{\mathcal{W}}\right.\right.$ such that $\lambda(] 0, \varepsilon[) \subset \mathcal{W}$ and $\lambda(0)=\left(p_{0}, \psi\right)$. We denote $\phi(s)=\left(y_{0}(s), y_{1}(s), \ldots, y_{n-1}(s), t_{1}(s), \ldots, t_{p}(s)\right)$ and $\psi(s)=\left(\psi_{1}(s), \ldots, \psi_{p}(s)\right)$. Since $F(\phi(s)) \equiv 0$, we have:

$$
0=\frac{d}{d s} F(\phi(s))=y_{0}^{\prime}(s) \frac{\partial F}{\partial y_{0}}(\phi(s))+\sum_{i=1}^{n-1} y_{i}^{\prime}(s) \frac{\partial F}{\partial y_{i}}(\phi(s))+\sum_{i=1}^{p} t_{i}^{\prime}(s) \frac{\partial F}{\partial t_{i}}(\phi(s)) .
$$

Multiplying by $\psi(s)$ we obtain:

$$
-y_{0}^{\prime}(s) \sum_{i=1}^{p} \psi_{i}(s) \frac{\partial F_{i}}{\partial y_{0}}(\phi(s))=\sum_{j=1}^{n-1} y_{j}^{\prime}(s) \sum_{i=1}^{p} \psi_{i}(s) \frac{\partial F_{i}}{\partial y_{j}}(\phi(s))+\sum_{j=1}^{p} t_{j}^{\prime}(s) \sum_{i=1}^{p} \psi_{i}(s) \frac{\partial F_{i}}{\partial t_{j}}(\phi(s)) .
$$

Since $\phi$ is analytic, thus bounded at $s=0$, by applying the Cauchy-Schwarz inequality one finds a constant $C>0$ such that:

$$
\begin{aligned}
& \mid\left(y_{0}^{\prime}(s) \sum_{i=1}^{p} \psi_{i}(s) \frac{\partial F_{i}}{\partial y_{0}}(\phi(s)) \mid \leq\right. \\
& \quad C\left\|\left(\sum_{i=1}^{p} \psi_{i} \frac{\partial F_{i}}{\partial y_{1}}(\phi), \ldots, \sum_{i=1}^{p} \psi_{i} \frac{\partial F_{i}}{\partial y_{n-1}}(\phi), \sum_{i=1}^{p} \psi_{i} \frac{\partial F_{i}}{\partial t_{1}}(\phi), \ldots, \sum_{i=1}^{p} \psi_{i} \frac{\partial F_{i}}{\partial t_{p}}(\phi)\right)(s)\right\| .
\end{aligned}
$$


We have $l:=\operatorname{ord}_{s} y_{0}^{\prime}(s) \geq 0$ and $\operatorname{ord}_{s} y_{0}(s)=l+1 \geq 1$ since $y_{0}(0)=0$, thus $\left|y_{0}(s) \sum_{i=1}^{p} \psi_{i}(s) \frac{\partial F_{i}}{\partial y_{0}}(\phi(s))\right| \ll\left|y_{0}^{\prime}(s) \sum_{i=1}^{p} \psi_{i}(s) \frac{\partial F_{i}}{\partial y_{0}}(\phi(s))\right|$, which, together with (9), gives:

$$
\begin{aligned}
&\left\|\sum_{i=1}^{p} \psi_{i}(s) y_{0}(s) \frac{\partial F_{i}}{\partial y_{0}}(\phi(s))\right\| \ll \\
&\left\|\left(\sum_{i=1}^{p} \psi_{i} \frac{\partial F_{i}}{\partial y_{1}}(\phi), \ldots, \sum_{i=1}^{p} \psi_{i} \frac{\partial F_{i}}{\partial y_{n-1}}(\phi), \sum_{i=1}^{p} \psi_{i} \frac{\partial F_{i}}{\partial t_{1}}(\phi), \ldots, \sum_{i=1}^{p} \psi_{i} \frac{\partial F_{i}}{\partial t_{p}}(\phi)\right)(s)\right\| .
\end{aligned}
$$

This contradicts our assumption that $(\phi(s), \psi(s)) \in \mathcal{W}$, for $s \in] 0, \varepsilon[$.

The equivalence $(\mathrm{b}) \Leftrightarrow(\mathrm{c})$ has been proved by Jelonek [Je1], [Je2] in a slightly more general setting.

\section{INTEGRAL CLOSURE INTERPRETATION OF $t$-REGULARITY AND Malgrange-GaFfney Condition}

In the setting of complex polynomial mappings $f: \mathbb{C}^{n} \rightarrow \mathbb{C}^{p}$, one observed that $t$ regularity has an integral closure interpretation, cf [Ti4, Remark 2.9] for $p=1$ and [Ga3] for $p \geq 1$. Actually Gaffney described in terms of the integral closure of modules a noncharacteristic condition for $p \geq 2$ [Ga3, Def.1] which turns out to be equivalent to the $t$-regularity. His technique works under the supplementary assumption that $f$ is "fair", see below. Let us first give the interpretation of the $t$-regularity of polynomial mappings $f: \mathbb{R}^{n} \rightarrow \mathbb{R}^{p}$ which are fair. We then prove the real counterpart of Gaffney's result [Ga3, Theorem 17].

4.1. Real integral closure of modules. Let us denote by $\mathcal{A}_{n}$ the local ring of real analytic function germs at the origin in $\mathbb{R}^{n}$ and by $\mathcal{A}_{n}^{p}$ the free $\mathcal{A}_{n}$-module of rank $p$. One denotes by $\mathcal{A}_{X, x}$ the local ring of real analytic function germs on the real analytic space germ $(X, x)$ and by $\mathcal{A}_{X, x}^{p}$ the free $\mathcal{A}_{X, x}$-module of rank $p$.

Definition 4.1. [Ga1, Def. 4.1] Let $(X, x) \subset\left(\mathbb{R}^{n}, x\right)$ be a real analytic germ and let $M$ be a $A_{X, x}$-submodule of $\mathcal{A}_{X, x}^{p}$. The real integral closure of $M$, denoted by $\bar{M}$, is the set of elements $h \in \mathcal{A}_{X, x}^{p}$ such that for any analytic path $\phi:(\mathbb{R}, 0) \rightarrow(X, x)$, we have $h \circ \phi \in \mathcal{A}_{1}\left(\phi^{*}(M)\right)$, where $\mathcal{A}_{1}\left(\phi^{*}(M)\right)$ denotes the $\mathcal{A}_{1}$-submodule of $\mathcal{A}_{1}^{p}$ generated by the elements $w \circ \phi, \forall w \in M$.

In the complex setting one has some equivalent definitions, see for example [Ga1, Prop. $1.7,1.11]$, which hold in the real setting except of [Ga1, Prop. 1.7] where it is necessary to assume that the regular points of $X$ are dense in $X$, see [Ga1, p. 318].

There is an interpretation of the conormal in terms of the integral closure of modules in the complex setting, see for instance [Ga2, GTW]. The next result was formulated in [Ga3, Prop. 4] in the complex setting but one can show that it holds over the reals if one assumes that the regular points of $X$ are dense, see [Di] for the details and generalization. Under the notations of $\S 2.1$ one has: 
Lemma 4.2. Let $(X, x) \subset\left(\mathbb{R}^{m}, x\right)$ be an equidimensional real analytic germ defined by a mapping germ $\tilde{F}:\left(\mathbb{R}^{m}, x\right) \rightarrow\left(\mathbb{R}^{p}, 0\right)$. Suppose that the regular points of $X$ are dense in $X$. Let $g:\left(\mathbb{R}^{m}, x\right) \rightarrow \mathbb{R}$ and denote $G:=(\tilde{F}, g)$. Let $V \subset \mathbb{R}^{m}$ be a linear subspace. Then the following are equivalent:

(a) There exist $H \in C_{g, x}(X)$ such that $H \supset V$.

(b) $\overline{J M_{X}(G)_{V}} \subsetneq \overline{J M_{X}(G)}$, where $J M_{X}(G)_{V}$ denotes the $\mathcal{A}_{X, x}$-submodule of $\mathcal{A}_{X, x}^{p+1}$ generated by $\left\{v_{1} \frac{\partial G}{\partial z_{1}}+\ldots+v_{m} \frac{\partial G}{\partial z_{m}} ; \forall v=\left(v_{1}, \ldots, v_{m}\right) \in V\right\}$, and $J M_{X}(G)$ denotes the $\mathcal{A}_{X, x}$-submodule of $\mathcal{A}_{X, x}^{p+1}$ generated by $\left\{\frac{\partial G}{\partial z_{1}}, \ldots, \frac{\partial G}{\partial z_{m}}\right\}$.

4.2. The "fair" condition. Let $f=\left(f_{1}, \ldots, f_{p}\right): \mathbb{R}^{n} \rightarrow \mathbb{R}^{p}$ be a polynomial mapping and let $\tilde{f}_{i}\left(x_{0}, x_{1}, \ldots, x_{n}\right)$ denote the homogenization of $f_{i}$ of degree $d_{i}:=\operatorname{deg} f_{i}$, for $1 \leqslant i \leqslant p$. Let:

$$
\mathcal{Z}=\bigcap_{i=1}^{p}\left\{\tilde{F}_{i}\left(x_{0}, x_{1}, \ldots, x_{n}, t_{1}, \ldots, t_{p}\right):=\tilde{f}_{i}\left(x_{0}, x_{1}, \ldots, x_{n}\right)-t_{i} x_{0}^{d_{i}}=0\right\} \subset \mathbb{P}^{n} \times \mathbb{R}^{p}
$$

and compare to the definition of $\mathbb{X}$ in $\S 2$ and to the notations in $\S 2.3$.

One notices the inclusion $\mathbb{X} \subset \mathcal{Z}$ and the equality $\mathbb{X} \backslash H^{\infty} \times \mathbb{R}^{p}=\mathcal{Z} \backslash H^{\infty} \times \mathbb{R}^{p}$. At any $p_{0} \in \mathbb{X}^{\infty}$ we may use the equations of $\mathcal{Z}$ in the open subspace $\mathcal{Z} \backslash H^{\infty} \times \mathbb{R}^{p} \simeq \mathbb{R}^{n}$. However, one does not have the equality $\mathbb{X}=\mathcal{Z}$ in general. Gaffney works in [Ga3] with the space $\mathcal{Z}$ assuming the equality $\mathcal{Z}=\mathbb{X}$ which he translates by " $f$ is fair" [Ga3, p. 158]. This is imposed by the theory of integral closure of modules since "fair" implies that the regular points of $\mathcal{Z}$ are dense in $\mathcal{Z}$ and that $\mathcal{Z}$ is equidimensional analytic. See Example 5.10 in which $\mathbb{X} \neq \mathcal{Z}$ and therefore this technique does not apply, whereas the statement and proof of the equivalence result Theorem 3.1 hold true.

4.3. $t$-regularity and fair polynomial mappings. Let $f=\left(f_{1}, \ldots, f_{p}\right): \mathbb{R}^{n} \rightarrow \mathbb{R}^{p}$ be a fair polynomial mapping. As in $\S 2.2$, we consider the charts $U_{j} \times \mathbb{R}^{p}$ of $\mathbb{P}^{n} \times \mathbb{R}^{p}$, where $U_{j}=\left\{x_{j} \neq 0\right\}, j=0,1, \ldots, n$ and we identify the chart $U_{0}$ with the affine space $\mathbb{R}^{n}$.

Let $p_{0} \in \mathbb{X}^{\infty}$. Up to some linear change of coordinate one may assume that $p_{0} \in$ $\mathbb{X}^{\infty} \cap\left(U_{n} \times \mathbb{R}^{p}\right)$. In the chart $U_{n} \times \mathbb{R}^{p}$, one considers the change of coordinates $y_{0}=$ $x_{0} / x_{n}, \ldots, y_{n-1}=x_{n-1} / x_{n}$. In this coordinate system and since $f$ is fair, one has

$$
\mathbb{X} \cap\left(U_{n} \times \mathbb{R}^{p}\right)=\bigcap_{i=1}^{p}\left\{\tilde{F}_{i}(y, t)=\tilde{f}_{i}\left(y_{0}, y_{1}, \ldots, y_{n-1}, 1\right)-t_{i} y_{0}^{d_{i}}=0\right\} .
$$

Proposition 4.3. Let $f: \mathbb{R}^{n} \rightarrow \mathbb{R}^{p}$ be a fair polynomial map. Then $f$ is t-regular at $p_{0} \in \mathbb{X}^{\infty}$ if and only if one of the following equivalent conditions is satisfied:

$$
\begin{gathered}
\partial \tilde{F} / \partial t_{i} \in \overline{\left\{\partial \tilde{F} / \partial y_{1}, \ldots, \partial \tilde{F} / \partial y_{n-1}\right\}}, \quad \forall i=1, \ldots, p . \\
\partial \tilde{F} / \partial t_{i} \in \overline{\left\{y_{0} \partial \tilde{F} / \partial y_{0}, \partial \tilde{F} / \partial y_{1}, \ldots, \partial \tilde{F} / \partial y_{n-1}\right\}}, \quad \forall i=1, \ldots, p .
\end{gathered}
$$


Proof. Let $V:=\mathbb{R}^{n} \times 0 \subset \mathbb{R}^{n} \times \mathbb{R}^{p}$. By Definitions 2.4 and 2.5, $p_{0}$ is a $t$-regular point if and only if there are no hyperplanes $H \supset V$ such that $H \in \mathcal{C}_{p_{0}}^{\infty}$. By Lemma 4.2 applied for $G=(\tilde{F}, g)$ and $V$, this is equivalent to the following:

$$
\frac{\partial G}{\partial t_{i}} \in \overline{\left\{\frac{\partial G}{\partial y_{0}}, \frac{\partial G}{\partial y_{1}}, \ldots, \frac{\partial G}{\partial y_{n-1}}\right\}} \text {, for } i=1, \ldots, p .
$$

By Definition 4.1, (12) means that for any $\phi:(\mathbb{R}, 0) \rightarrow\left(\mathbb{X}, p_{0}\right)$, there exist $\lambda_{0}, \lambda_{1}, \ldots$, $\lambda_{n-1} \in \mathcal{A}_{1}$ such that:

$$
\left(\begin{array}{c}
\frac{\partial \tilde{F}}{\partial t_{i}}(\phi(s)) \\
0
\end{array}\right)=\lambda_{0}(s)\left(\begin{array}{c}
\frac{\partial \tilde{F}}{\partial y_{0}}(\phi(s)) \\
1
\end{array}\right)+\lambda_{1}(s)\left(\begin{array}{c}
\frac{\partial \tilde{F}}{\partial y_{1}}(\phi(s)) \\
0
\end{array}\right)+\cdots+\lambda_{n-1}(s)\left(\begin{array}{c}
\frac{\partial \tilde{F}}{\partial y_{n-1}}(\phi(s)) \\
0
\end{array}\right)
$$

which is in turn equivalent to (10).

That (10) implies (11) is obvious. The converse is the same as Gaffney's proof of [Ga3], holds over the reals too and is based on Parusinski's proof [Pa1, Lemma 3.2].

Theorem 4.4. A fair polynomial mapping $f: \mathbb{R}^{n} \rightarrow \mathbb{R}^{p}$ satisfies the Malgrange-Gaffney condition at $p_{0} \in \mathbb{X}^{\infty}$ if and only if $f$ is t-regular at this point.

Proof. That $f$ is $t$-regular at $p_{0}$ is equivalent to (11) of Proposition 4.3. In turn, by [Ga1, Prop. (1.7)] which hold over $\mathbb{R}$ too since $f$ is fair, see [Ga1, p. 318] and [Di], this is equivalent to:

$$
y_{0}^{d_{j}} M_{J}(j, \tilde{F}) \in \overline{\left\langle M_{I}(\tilde{F})\right\rangle}, j=1, \ldots, p,
$$

where $\left\langle M_{I}(\tilde{F})\right\rangle$ denotes the ideal generated by the $p \times p$ minors of the matrix whose columns are $\left(y_{0} \partial \tilde{F} / \partial y_{0}, \partial \tilde{F} / \partial y_{1}, \ldots, \partial \tilde{F} / \partial y_{n-1}\right)$, and $M_{J}(j, \tilde{F})$ is a maximal minor of the same matrix with the $j$ th row deleted.

Using [Ga1, Prop 4.2], one has that (13) is equivalent to the existence of $C>0$ such that:

$$
\sup _{J, j}\left\|y_{0}^{d_{j}}\right\|\left\|M_{J}(j, \tilde{F})(y, t)\right\| \leq C \sup _{I}\left\|M_{I}(\tilde{F})(y, t)\right\| \text {. }
$$

Dividing both sides by $\left\|y_{0}^{k}\right\|$, where $k=\sum_{l=1}^{p}\left(d_{l}-1\right)$, and using properties of determinant and the following relations between the partials of $f$ and the partials of $\tilde{F}$ :

$$
\begin{cases}\frac{\partial \tilde{F}_{j}}{\partial y_{i}} / y_{0}^{d_{j}-1}=\frac{\partial f_{j}}{\partial x_{i}}, & 1 \leq i \leq n-1,1 \leq j \leq p \\ \frac{\partial \tilde{F}_{j}}{\partial t_{l}}=-y_{0}^{d_{j}} \delta_{l, j}, & 1 \leq j, l \leq p \\ \frac{\partial \tilde{F}_{j}}{\partial y_{0}} / y_{0}^{d_{j}-1}=-\left(x_{1} \frac{\partial f_{j}}{\partial x_{1}}+\ldots+x_{n} \frac{\partial f_{j}}{\partial x_{n}}\right), & 1 \leq j \leq p\end{cases}
$$

we obtain

$$
\sup _{J, j}\left\|1 / x_{n}\right\|\left\|M_{J}^{\prime}(j, f)(x)\right\| \leq C \sup _{I}\left\|M_{I}^{\prime}(f)(x)\right\|,
$$

where the $M^{\prime}$ matrices are defined as follows. If $I=\left(i_{1}<\ldots<i_{p}\right)$ with $i_{1} \neq 1$, i.e., if the matrix indexed by $I$ does not contain the column $y_{0} \partial \tilde{F} / \partial y_{0}$ then $M_{I}^{\prime}(f)=M_{I}(f)$, 
and otherwise, one replaces the column vector with the $\partial f / \partial x_{n}$ terms by $\sum_{l=1}^{n} \frac{x_{l}}{x_{n}} \partial f / \partial x_{l}$. A similar substitution should be made to define the $M_{J}^{\prime}(j, f)$ terms.

Using the fact that $\left\|x_{n}\right\| \geq\left\|x_{j}\right\|$, for $j=1, \ldots, n$, the inequality (14) is equivalent to generalized Malgrange-Gaffney condition (1).

REMARK 4.5. Jelonek [Je2, Je1] proved directly that the Malgrange-Gaffney condition (actually in a more general form) is equivalent to the $K O S$-regularity, by comparing the corresponding functions to the Kuo distance function $[\mathrm{Ku}]$.

\section{Atypical VAlues AND Fibrations at infinity}

Definition 5.1. Let $X \subset \mathbb{R}^{n}$ be a submanifold and let $f: X \rightarrow \mathbb{R}^{p}$ be a $C^{1}$-mapping, where $p<\operatorname{dim} X$. One says that $t_{0} \in \mathbb{R}^{p}$ is a typical value of $f$ if there exists a disk $D \subset \mathbb{R}^{p}$ centered at $t_{0}$ such that the restriction $f_{\mid}: f^{-1}(D) \rightarrow D$ is a topologically trivial fibration; otherwise one says that $t_{0}$ is atypical. We denote by $B(f)$ the set of atypical values of $f$ (or the bifurcation locus of $f$ ).

Note that by definition $B(f) \supset \overline{\operatorname{Im} f} \backslash \operatorname{Im} f$ and that the fibres of $f$ depend on the connected components of $\mathbb{R}^{p} \backslash B(f)$.

5.1. $\rho$-regularity. Let $K \subset \mathbb{R}^{n}$ be some compact (eventually empty) set and let $\rho$ : $\mathbb{R}^{n} \backslash K \rightarrow \mathbb{R}_{\geq 0}$ be a proper analytic submersion. Let $f: X \rightarrow \mathbb{R}^{p}$ be a $C^{1}$-mapping, where $X \subset \mathbb{R}^{n}$ is a submanifold.

Definition 5.2 ( $\rho$-regularity at infinity). We say that $f$ is $\rho$-regular at $p_{0} \in \mathbb{X}^{\infty}$ if there is an open neighborhood $U \subset \mathbb{P}^{n} \times \mathbb{R}^{p}$ of $p_{0}$ and an open neighborhood $D \subset \mathbb{R}^{p}$ of $\tau\left(p_{0}\right)$ such that, for all $t \in D$, the fibre $f^{-1}(t) \cap U$ intersects all the levels of the restriction $\rho_{\mid U \cap \mathbb{R}^{n}}$ and this intersection is transversal.

We call Milnor set the critical locus $\operatorname{Sing}(f, \rho)$ and we denote it by $\mathcal{M}(f)$.

We say that the fibre $f^{-1}\left(t_{0}\right)$ is $\rho$-regular at infinity if $f$ is $\rho$-regular at all points $p_{0} \in \mathbb{X}^{\infty} \cap \tau^{-1}\left(t_{0}\right)$. We call:

$$
S(f):=\left\{t_{0} \in \mathbb{R}^{p} \mid \exists\left\{\mathrm{x}_{j}\right\}_{j \in \mathbb{N}} \subset \mathcal{M}(f), \lim _{j \rightarrow \infty}\left\|\mathrm{x}_{j}\right\|=\infty \text { and } \lim _{j \rightarrow \infty} f\left(\mathrm{x}_{j}\right)=t_{0}\right\} .
$$

the set of asymptotic $\rho$-nonregular values. We denote $A_{\rho}:=f(\operatorname{Sing} f) \cup S(f)$ and call it the $\rho$-bifurcation set.

REMARK 5.3. The definition of $\rho$-regularity at infinity of a fibre $f^{-1}\left(t_{0}\right)$ does not depend on any proper extension of $f$, since it is equivalent to the following: for any sequence $\left\{\mathrm{x}_{k}\right\}_{k \in \mathbb{N}} \subset \mathbb{R}^{n},\left\|\mathrm{x}_{k}\right\| \rightarrow \infty, f\left(\mathrm{x}_{k}\right) \rightarrow t_{0}$, there exists some $k_{0}=k_{0}\left(\left\{\mathrm{x}_{k}\right\}_{k \in \mathbb{N}}\right)$ such that, if $k \geq k_{0}$ then $f$ is transversal to $\rho$ at $\mathrm{x}_{k}$. It also follows from the definition that if $f^{-1}\left(t_{0}\right)$ is $\rho$-regular at infinity then this fibre has at most isolated singularities.

The transversality of the fibres of $f$ to the levels of $\rho$ is a "Milnor type" condition. In case $\rho$ is the Euclidean norm, denoted in this paper by $\rho_{E}$, this condition has been used by John Milnor in the local study of singular functions [Mi, §4,5]. For complex polynomial functions, transversality to big spheres (i.e. $\rho_{E}$-regularity, in our definition) was used in [Br, pag. 229] and later in [NZ], where it is called M-tameness. The name "Milnor set" 
occurs in [NZ] too. Distance functions like $\rho$ are also central ingredients in defining regular stratifications, e.g. Mather [Ma], Kuo [Ku], Bekka [Be].

ExAmple 5.4. Let $\rho: \mathbb{R}^{n} \rightarrow \mathbb{R}_{\geq 0}, \rho(x)=\left(\sum_{i=1}^{n}\left|x_{i}\right|^{2 p_{i}}\right)^{1 / 2 p}$, where $\left(w_{1}, \ldots, w_{n}\right) \in \mathbb{N}^{n}$, $p=\operatorname{lcm}\left\{w_{1}, \ldots, w_{n}\right\}$ and $w_{i} p_{i}=p, \forall i$. This function is "adapted" to polynomials which are quasihomogeneous of type $\left(w_{1}, \ldots, w_{n}\right)$. By using it, one can show that a value $c \in \mathbb{R}$ is atypical for such a polynomial if and only if $c$ is a critical value of $f$ (hence only the value 0 can be atypical). Namely, let $E_{r}:=\left\{x \in \mathbb{R}^{n} \mid \rho(x)<r\right\}$ for some $r>0$. Then the local Milnor fibre of $f$ at $0 \in \mathbb{R}^{n}$ (i.e. $f^{-1}(c) \cap E_{\varepsilon}$, for some small enough $\varepsilon$ and $0<|c| \ll \varepsilon)$ is diffeomorphic to the global fibre $f^{-1}(c)$, since $f^{-1}(c)$ is transversal to $\partial \overline{E_{r}}$, $\forall r \geq \varepsilon$.

\section{2. $t$-regularity implies $\rho_{E}$-regularity.}

Proposition 5.5. Let $X \subset \mathbb{R}^{n}$ be semi-algebraic and let $f: X \rightarrow \mathbb{R}^{p}$ be a nontrivial semialgebraic $C^{1}$ mapping, where $n>p$. If $f$ is t-regular at $p_{0} \in \mathbb{X}^{\infty}$ then $f$ is $\rho_{E}$-regular at $p_{0}$.

Proof. We may assume without loss of generality that $p_{0}=([0: 0: \ldots: 1], 0, \ldots 0)$.

Let $d^{\infty}: \mathbb{X} \cap U_{n} \times \mathbb{R}^{p} \rightarrow \mathbb{R}_{\geq 0},(y, t) \mapsto \frac{y_{0}^{2}}{y_{1}^{2}+\ldots+y_{n-1}^{2}+1}$ and note that $d^{\infty}(y, t)=\frac{1}{\rho_{E}^{2}(\mathrm{x})}$. As usual, we denote by $g$ the projection to the variable $y_{0}$. At $p_{0}$, the functions $g^{2}$ and $d^{\infty}$ differ by a unit, they have the same zero locus $\mathbb{X}^{\infty}$ and the same levels. Therefore $\mathcal{C}_{p_{0}}^{\infty}=C_{g, p_{0}}\left(\mathbb{X} \backslash \mathbb{X}^{\infty} \cap\left(U_{n} \times \mathbb{R}^{p}\right)\right)=C_{g^{2}, p_{0}}\left(\mathbb{X} \backslash \mathbb{X}^{\infty} \cap\left(U_{n} \times \mathbb{R}^{p}\right)\right)=C_{d^{\infty}, p_{0}}\left(\mathbb{X} \backslash \mathbb{X}^{\infty} \cap\left(U_{i} \times \mathbb{R}^{p}\right)\right)$, where the last equality follows by Lemma 2.3 .

The $t$-regularity at $p$ (Definition 2.5) is therefore equivalent to:

$$
C_{\tau}\left(\mathbb{P}^{n} \times \mathbb{R}^{p}\right) \cap C_{d^{\infty}, p_{0}}\left(\mathbb{X} \backslash \mathbb{X}^{\infty} \cap U_{i} \times \mathbb{R}^{p}\right)=\emptyset
$$

which implies that, in some neighbourhood of $p_{0}$ intersected with $\mathbb{R}^{n}$, the fibres $\{\tau=$ const.\} are transverse to the levels of the function $d^{\infty}$, which coincide with the levels of the function $\rho_{E}$.

REMARK 5.6. The converse of Proposition 5.5 is not true, see Example 5.9.

We have the following general structure result:

Theorem 5.7. Let $f: X \rightarrow \mathbb{R}^{p}$ be a $C^{2}$-mapping on a submanifold $X \subset \mathbb{R}^{n}$, where $\operatorname{dim} X>p>0$. Then:

(a) (Fibration theorem)

$S(f)$ is a closed set. If $X$ is moreover closed, then $A_{\rho_{E}}:=f(\operatorname{Sing} f) \cup S(f)$ is a closed set and the restriction:

$$
f_{\mid}: X \backslash f^{-1}\left(A_{\rho_{E}}\right) \rightarrow \mathbb{R}^{p} \backslash A_{\rho_{E}}
$$

is a locally trivial fibration over each connected component of $\mathbb{R}^{p} \backslash\left(A_{\rho_{E}}\right)$.

In particular $B(f) \subset A_{\rho_{E}}$.

(b) (Semi-algebraic Morse-Sard theorem for asymptotic non-regular values) Assume that $X$ is semi-algebraic and that $f$ is a semi-algebraic mapping. Then $S(f)$ and $A_{\rho_{E}}$ are semi-algebraic sets of dimension $\leq p-1$. 
By chaining Theorem 3.1 to Proposition 5.5, we get:

Corollary 5.8. Let $f: \mathbb{R}^{n} \rightarrow \mathbb{R}^{p}$ be a nontrivial semi-algebraic $C^{2}$ mapping. If $f$ is $K O S$-regular at $p_{0} \in \mathbb{X}^{\infty}$ then $f$ is $\rho_{E}$-regular at $p_{0}$. In particular, one has the inclusions:

$$
S(f) \subset K_{\infty}(f) \text { and } A_{\rho_{E}} \subset A_{K O S} \text {. }
$$

The above results prove that Theorem 5.7 extends the main result of [KOS], and this extension turns out to be strict, as showed by the next example.

ExAmple 5.9. [PZ] The polynomials $f_{n, q}: \mathbb{C}^{3} \rightarrow \mathbb{C}, f_{n, q}(x, y, z):=x-3 x^{2 n+1} y^{2 q}+$ $2 x^{3 n+1} y^{3 q}+y z$, where $n, q \in \mathbb{N} \backslash\{0\}$, are $\rho_{E}$-regular at infinity, more precisely $S\left(f_{n . q}\right)=\emptyset$. Then it is shown in [PZ] that $f_{n, q}$ satisfies Malgrange's condition (hence it is $t$-regular at infinity) for any $t \in \mathbb{C}$ if and only if $n \leq q$. For $n>q$ we therefore get $\emptyset=S\left(f_{n . q}\right) \subsetneq$ $K_{\infty}\left(f_{n . q}\right) \neq \emptyset$. In particular, for $n>q$ the polynomial is $\rho$-regular but not $t$-regular.

EXAmple 5.10. Let $f: \mathbb{R}^{3} \rightarrow \mathbb{R}^{2}, f(x, y, z)=\left(x^{2}, x y\right)$. We have $\mathbb{X}^{\infty}=A \cup B$, where $A=\left\{\left([0: 0: c: d],\left(0, t_{2}\right)\right) \in \mathbb{P}^{3} \times \mathbb{R}^{2}\right\}$ and $B=\left\{\left([0: 0: 0: 1],\left(t_{1}, t_{2}\right)\right) \in \mathbb{P}^{3} \times \mathbb{R}^{2} \mid t_{1}>0\right\}$, and that $\mathcal{Z}^{\infty}:=\mathcal{Z} \cap\left(H^{\infty} \times \mathbb{R}^{2}\right)=\left\{\left([0: 0: y: z],\left(t_{1}, t_{2}\right)\right) \in \mathbb{P}^{3} \times \mathbb{R}^{2}\right\}$. Thus $\mathbb{X}^{\infty} \subsetneq \mathcal{Z}^{\infty}$ and by straightforward computations we get that $f$ is not "fair" in the sense of Gaffney at any point $p_{0} \in \mathbb{X}^{\infty}$. Therefore one cannot use Gaffney's approach for $f$. Nevertheless, we still have the equivalence of $t$-regularity with the Malgrange-Gaffney condition (Theorem 3.1) and the fibration theorem (Theorem 5.7), see also Figure 1.

By straightforward computations one gets $f(\operatorname{Sing} f)=\{(0,0)\}$ and $B(f)=K(f)=$ $S(f)=\tau(A)=\left\{\left(0, t_{2}\right) ; t_{2} \in \mathbb{R}\right\}$.

\section{Proof of Theorem 5.7}

6.1. Proof of (b). The image $f(\operatorname{Sing} f)$ by $f$ of the semi-algebraic set Sing $f$ is semialgebraic, by the Tarski-Seidenberg theorem, and of dimension $\leq p-1$ by the semialgebraic Sard theorem.

To show that $S(f)$ is semi-algebraic, we use the semi-algebraic embedding $\varphi: \mathbb{R}^{n} \rightarrow$ $\mathbb{R}^{n+1} \times \mathbb{R}^{p},\left(x_{1}, \ldots, x_{n}\right) \mapsto\left(\frac{1}{\sqrt{1+\|\mathrm{x}\|^{2}}}, \frac{x_{1}}{\sqrt{1+\|\mathrm{x}\|^{2}}}, \ldots, \frac{x_{n}}{\sqrt{1+\|\mathrm{x}\|^{2}}}, f(\mathrm{x})\right)$. Let $V_{1}:=\overline{\varphi(\mathcal{M}(f))} \cap$ $\left\{\left(z_{0}, z_{1}, \ldots, z_{n}, t\right) \in \mathbb{R}^{n+1} \times \mathbb{R}^{p} \mid z_{0}=0\right\}$ and let $\pi: \mathbb{R}^{n+1} \times \mathbb{R}^{p} \rightarrow \mathbb{R}^{p}$ be the canonical projection. Then $V_{1}$ is semi-algebraic and $S(f)=\pi\left(V_{1}\right)$, so we may conclude by the Tarski-Seidenberg theorem.

To prove the dimension assertion for $S(f)$ we need some preliminaries.

Partial Thom stratification at infinity. We show that $\mathbb{X}^{\infty}$ may be endowed with a stratification having good enough properties such that one may use it to define the stratified singular locus of $\tau_{\mid \mathbb{X}^{\infty}}$. By "stratifications" we mean, as usual, locally finite stratifications satisfying the frontier condition. For some strata $A, B$, we write $B \prec A$ to say that $B \subset \bar{A} \backslash A$.

Proposition 6.1. Let $f: X \rightarrow \mathbb{R}^{p}$ be a semi-algebraic $C^{1}$-mapping on a semi-algebraic subset $X \subset \mathbb{R}^{n}$. There exists a semi-algebraic Whitney (a)-regular stratification $\mathcal{S}$ of $\mathbb{X}$ 
such that $\mathbb{X}^{\infty}$ is a union of strata, and that any pair of strata $B \prec A$, with $A \subset \mathbb{X} \backslash \mathbb{X}$ and $B \subset \mathbb{X}^{\infty}$, satisfy the Thom $\left(a_{g}\right)$-regularity condition with respect to some function $g$ defining locally $\mathbb{X}^{\infty}$ in $\mathbb{X}$.

Proof. We follow [Ti2, §2] and start with some Whitney (a)-regular stratification of $\mathbb{X}$ with semi-algebraic strata; this exists after Whitney [Wh], see also [GWPL, Ch. I]. One then refines it to a semi-algebraic stratification such that $\mathbb{X}^{\infty}$ is a union of strata, see [GWPL, Ch. I]. Next, since the $\left(a_{g}\right)$-regularity condition is stratifiable (see e.g. [GWPL, Ch. I], [DŁS], [Be, §3]), applying the Thom condition to the pairs of strata as in the above statement yields a further refinement which is the desired stratification $\mathcal{S}$, at least locally.

We however need to show that this refinement is a globally defined stratification of $\mathbb{X}^{\infty}$. The argument goes as follows: in the $\left(a_{g}\right)$-regularity test one uses the limits at some point of $\mathbb{X}^{\infty}$ of the tangent hyperplanes along strata coming from $\mathbb{X} \backslash \mathbb{X}$. These limits are precisely described by the space of characteristic covectors at infinity $\mathcal{C}^{\infty}$. But by Lemma $2.3, \mathcal{C}^{\infty}$ is independent of the function $g$ defining $\mathbb{X}^{\infty}$ locally.

Definition 6.2. One calls partial Thom stratification at infinity a stratification $\mathcal{S}$ as in Proposition 6.1.

Such stratifications ${ }^{1}$ depend of course on the embedding $X \subset \mathbb{R}^{n}$.

With these notations and definitions, let $\mathcal{S}=\left\{S_{i}\right\}_{i \in I}$ be a semi-algebraic partial Thom stratification at infinity, the existence of which has been proved above. Consider the projection $\tau: \mathbb{P}^{n} \times \mathbb{R}^{p} \rightarrow \mathbb{R}^{p}$ and $t_{0} \in \mathbb{R}^{p}$. The critical locus at infinity of the restriction $\tau_{\mid \mathbb{X}}$ with respect to $\mathcal{S}$ is defined as follows:

$$
\operatorname{Sing}_{\mathcal{S}}^{\infty} \tau_{\mid \mathbb{X}}:=\bigcup_{\mathcal{S}_{i} \subset \mathbb{X}}^{\infty} \operatorname{Sing} \tau_{\mid \mathcal{S}_{i}}
$$

Since the stratification of $\mathbb{X}^{\infty}$ is in particular Whitney (a)-regular, it follows that $\operatorname{Sing}_{\mathcal{S}}^{\infty} \tau_{\mid \mathbb{X}}$ is a closed semi-algebraic subset of $\mathbb{X}^{\infty}$. Then, by the semi-algebraic Sard theorem, the image $\tau\left(\operatorname{Sing}_{\mathcal{S}}^{\infty} \tau_{\mid \mathbb{X}}\right) \subset \mathbb{R}^{p}$ is semi-algebraic and of dimension $\leq p-1$.

It also follows from the definition that:

$$
p_{0} \notin \operatorname{Sing}_{\mathcal{S}}^{\infty} \tau_{\mid \mathbb{X}} \Longrightarrow f \text { is } t \text {-regular at } p_{0},
$$

which implies that $\mathbb{R}^{p} \backslash \tau\left(\operatorname{Sing}_{\mathcal{S}}^{\infty} \tau_{\mid \mathbb{X}}\right)$ is included in the set of $t$-regular values of $f$. Therefore, by Proposition 5.5, we get the inclusion $\mathbb{R}^{p} \backslash \tau\left(\operatorname{Sing}_{\mathcal{S}}^{\infty} \tau_{\mid \mathbb{X}}\right) \subset \mathbb{R}^{p} \backslash S(f)$, which shows that $\operatorname{dim} S(f) \leq p-1$.

6.2. Proof of (a). Let $t_{0} \in \overline{S(f)}$ and let $\left\{t_{i}\right\}_{i \in \mathbb{N}} \subset S(f)$ be a sequence such that $\lim _{i \rightarrow \infty} t_{i}=t_{0}$. By definition, for every $i$ we have a sequence $\left\{\mathrm{x}_{i, k}\right\}_{k \in \mathbb{N}} \subset \mathcal{M}(f)$ such that $\lim _{k \rightarrow \infty}\left\|\mathrm{x}_{i, k}\right\|=\infty$ and $\lim _{k \rightarrow \infty} f\left(\mathrm{x}_{i, k}\right)=t_{i}$. For each $i$, there exists $k(i) \in \mathbb{N}$ such that if $k \geqslant k(i)$ then $\left\|\mathrm{x}_{i, k}\right\|>i$ and $\left|f\left(\mathrm{x}_{i, k}\right)-t_{i}\right|<1 / i$. Setting $\mathrm{x}_{i}:=\mathrm{x}_{i, k(i)}$, one gets a sequence $\left\{\mathrm{x}_{i}\right\}_{i \in \mathbb{N}} \subset \mathcal{M}(f)$ such that $\lim _{i \rightarrow \infty}\left\|\mathrm{x}_{i}\right\|=\infty$ and $\lim _{i \rightarrow \infty} f\left(\mathrm{x}_{i}\right)=t_{0}$. This shows that $t_{0} \in S(f)$, hence $S(f)$ is closed.

\footnotetext{
${ }^{1}$ have been introduced in [Ti2, Def. 2.1], [Ti5, Appendix 1], for $p=1$.
} 
Let us assume now that $X$ is closed and let $t_{0} \in \overline{f(\operatorname{Sing} f)} \cup S(f)$. We may assume that $t_{0} \in \overline{f(\operatorname{Sing} f)}$ since we have just proved that $S(f)$ is closed. Then there exists a sequence $\left\{\mathrm{x}_{j}\right\}_{j \in \mathbb{N}} \subset \operatorname{Sing} f$, such that $\lim _{j \rightarrow \infty} f\left(\mathrm{x}_{j}\right)=t_{0}$. If $\left\{\mathrm{x}_{j}\right\}_{j \in \mathbb{N}}$ is non-bounded, we may choose a subsequence $\left\{\mathrm{x}_{j_{k}}\right\}_{k \in \mathbb{N}}$ such that $\lim _{k \rightarrow \infty}\left\|\mathrm{x}_{j_{k}}\right\|=\infty$ and $\lim _{k \rightarrow \infty} f\left(\mathrm{x}_{j_{k}}\right)=t_{0}$. Since Sing $f \subset \mathcal{M}(f)$, it follows that $t_{0} \in S(f)$ which is closed. If $\left\{\mathrm{x}_{j}\right\}_{j \in \mathbb{N}}$ is bounded, then we may choose a subsequence $\left\{\mathrm{x}_{j_{k}}\right\}_{k \in \mathbb{N}}$ such that $\lim _{k \rightarrow \infty} \mathrm{x}_{j_{k}}=\mathrm{x}_{0} \in X$ since $X$ is assumed to be closed, and that $\lim _{k \rightarrow \infty} f\left(\mathrm{x}_{j_{k}}\right)=t_{0}$. Since $\operatorname{Sing} f$ is a closed set, this implies $\mathrm{x}_{0} \in \operatorname{Sing} f$, and we get $t_{0}=f\left(\mathrm{x}_{0}\right) \in f(\operatorname{Sing} f)$, which shows that $t_{0} \in f(\operatorname{Sing} f) \cup S(f)$.

Let us finally show the fibration statement. We first prove a fibration result in the neighbourhood of infinity.

\section{Definition 6.3. (Topological triviality at infinity)}

We say that $f$ is topologically trivial at infinity at the value $t_{0} \in \mathbb{R}^{p}$ if there exists a compact set $K \subset \mathbb{R}^{n}$ and a ball $B_{\delta} \subset \mathbb{R}^{p}$ centered at $t_{0}$ such that the restriction:

$$
f_{\mid}:(X \backslash K) \cap f^{-1}\left(B_{\delta}\right) \rightarrow B_{\delta}
$$

is a trivial topological fibration.

Note that one may have two situations in which the mapping (17) may be a trivial fibration, namely whenever $B_{\delta} \subset \operatorname{Im} f$ or when $B_{\delta} \subset \mathbb{R}^{p} \backslash \operatorname{Im} f$. In the later, the fibration has empty fibre.

Proposition 6.4. ( $\rho$-regularity implies topological triviality at infinity) Let $f: X \rightarrow \mathbb{R}^{p}$ be a $C^{2}$ mapping, for $n>p$. If the fibre $f^{-1}\left(t_{0}\right)$ is $\rho$-regular at infinity, then $f$ is topologically trivial at infinity at $t_{0}$.

In particular, $f$ is topologically trivial at infinity at any value of $\mathbb{R}^{p} \backslash S(f)$.

Proof. Let $t_{0} \notin S(f)$. Since $S(f)$ is a closed set (as proved just above), there exists a closed ball $D$ centered at $t_{0}$ and included in $\mathbb{R}^{p} \backslash S(f)$. Then there exists some large enough radius $R_{0} \gg 0$ such that:

$$
\mathcal{M}(f) \cap f^{-1}(D) \backslash B_{R_{0}}^{n}=\emptyset .
$$

Indeed, if this were not true, then there exists a sequence $\left\{\mathrm{x}_{k}\right\}_{k \in \mathbb{N}} \subset f^{-1}(D) \cap \mathcal{M}(f)$ with $\lim _{k \rightarrow \infty}\left\|\mathrm{x}_{k}\right\|=\infty$ and since $D$ is compact, one may extract a sub-sequence $\left\{\mathrm{x}_{k_{i}}\right\}_{i \in \mathbb{N}} \subset$ $\mathcal{M}(f)$ with $\lim _{k \rightarrow \infty} f\left(\mathrm{x}_{k_{i}}\right)=t \in D$, which gives a contradiction to $D \cap S(f)=\emptyset$.

To prove the topological triviality at infinity at $t_{0}$ it is enough to show that the mapping:

$$
f_{\mid}: f^{-1}(D) \backslash B_{R}^{n} \rightarrow D
$$

is a trivial fibration on the manifold with boundary $\left(f^{-1}(D) \backslash B_{R}^{n}, f^{-1}(D) \cap S_{R}^{n-1}\right)$, for any $R \geq R_{0}$. This is a submersion by (18) but it is not proper, so one cannot apply Ehresmann's theorem directly. Instead, we consider the map $(f, \rho): f^{-1}(D) \backslash B_{R}^{n} \rightarrow$ $D \times[R, \infty[$. Now, as a direct consequence of its definition, this is a proper map. It is still a submersion by $(18)$ and since $\operatorname{Sing}(f, \rho)=\mathcal{M}(f)$. We then apply Ehresmann's theorem to the mapping $(f, \rho)$ in order to conclude that it is a locally trivial fibration, hence trivial over $D \times[R, \infty[$. Take now the projection $\pi: D \times[R, \infty[\rightarrow D$ which is a trivial fibration by definition and remark that our map (19) is the composition $\pi \circ(f, \rho)$ of two trivial fibrations, hence a trivial fibration too. 
REMARK 6.5. It is interesting to point out that the implication in the above proposition is not an equivalence in general. The paper [TZ] presents an example of a polynomial mapping $f: \mathbb{R}^{2} \rightarrow \mathbb{R}$ which is not $\rho_{E}$-regular at infinity at the value 0 , thus not $K O S$ regular either, but it is $C^{\infty}$ trivial at infinity at 0 . It is easier to give such examples in the topological cathegory, for instance $f(x, y)=x^{3}$ which is topologically equivalent to the projection on $x$, whereas $S(f)=\{0\}$.

We now complete the proof of Theorem 5.7(a). Since $\mathbb{R}^{p} \backslash(f(\operatorname{Sing} f) \cup S(f))$ is an open set, for any fixed $t_{0} \notin f(\operatorname{Sing} f) \cup S(f)$ there exists a closed ball $D$ centered at $t_{0}$ such that $D \subset \mathbb{R}^{p} \backslash f(\operatorname{Sing} f) \cup S(f)$. By the above proof of Proposition 6.4 and using the same notations, one has the trivial fibration (19) on the manifold with boundary $\left(f^{-1}(D) \backslash B_{R}^{n}, f^{-1}(D) \cap S_{R}^{n-1}\right)$, for any $R \geq R_{0}$.

Next, since $D \cap f(\operatorname{Sing} f)=\emptyset$, the restriction:

$$
f_{\mid}: f^{-1}(D) \cap \bar{B}_{R_{0}}^{n} \rightarrow D
$$

is a proper submersion on the manifold with boundary $\left(f^{-1}(D) \cap \bar{B}_{R_{0}}^{n}, f^{-1}(D) \cap S_{R_{0}}^{n-1}\right)$ and therefore a locally trivial fibration by Ehresmann's theorem, hence a trivial fibration over $D$.

We finally glue together the two trivial fibrations (20) and (19) by using an isotopy and the trivial fibration from the following commutative diagram, for some $R>R_{0}$ :

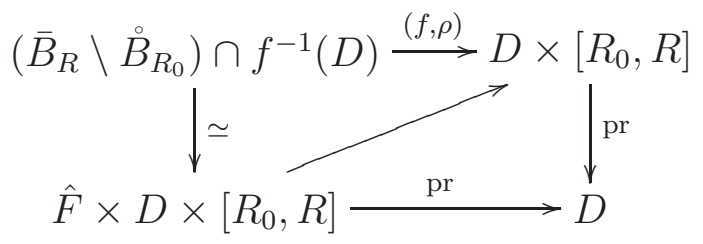

where $\hat{F}$ denotes the fibre of the trivial fibration $f_{\mid}: S_{R} \cap f^{-1}(D) \rightarrow D$ and does not depend on the radius $R>R_{0}$.

REMARK 6.6. If in Theorem 5.7(a) we do not assume that $X$ is closed then the fibration assertion holds if one replaces in the statement $f(\operatorname{Sing} f)$ by its closure $\overline{f(\operatorname{Sing} f)}$.

\section{REFERENCES}

[Be] K. BekKA, Regular stratification of subanalytic sets. Bull. London Math. Soc. 25 (1993), no. 1, 7-16.

[Br] S.A. Broughton, Milnor number and the topology of polynomial hypersurfaces, Inventiones Math. 92 (1988), 217-241.

[DŁS] Z. Denkowska, S. Łojasiewicz, J. Stasica, Certaines propriétés élémentaires des ensembles sous-analytiques. Bull. Acad. Polon. Sci. Sér. Sci. Math. 27 (1979), no. 7-8, 529-536 (1980).

[Di] L.R.G. Dias, PhD Thesis, Universidade de São Paulo and Université Lille 1.

[Du] A.H. Durfee, Five definitions of critical point at infinity. Singularities (Oberwolfach, 1996), 345-360, Progr. Math., 162, Birkhäuser, Basel, 1998.

[Ga1] T. GaffneY, Integral closure of modules and Whitney equisingularity, Inventiones Math. 107 (1992), 301-322.

[Ga2] T. Gaffney, Aureoles and integral closure of modules, Stratifications, Singularities and Differential Equations II, Travaux en Cours, 55. Hermann, Paris, 1997, 55-62. 
[Ga3] T. GAFFney, Fibers of polynomial mappings at infinity and a generalized Malgrange condition, Compositio Math. 119 (1999), p. 157-167.

[GTW] T. Gaffney, D. Trotman, L. Wilson, Equisingularity of sections, $t^{r}$ condition, and the integral closure of modules, J. Algebraic Geometry 18 (2009), p. 651-689.

[GWPL] C.G. Gibson, K. Wirthmüller, A.A. Du Plessis, E.J.N. Looijenga, Topological Stability of Smooth Mappings, Lect. Notes in Math. 552, Springer Verlag 1976.

[HaLe] HÀ H.V., Lê D.T., Sur la topologie des polynômes complexes, Acta Math. Vietnam. 9 (1984), no. 1, 21-32 (1985).

[Hi] H. Hironaka, Stratifications and flattness, in: Real and Complex Singularities, Oslo 1976, Sijhoff en Norhoff, Alphen a.d. Rijn 1977.

[Je1] Z. JeloneK, On the generalized critical values of a polynomial mapping. Manuscripta Math. 110 (2003), no. 2, 145-157.

[Je2] Z. JELONEK, On asymptotic critical values and the Rabier theorem. Geometric singularity theory, 125-133, Banach Center Publ., 65, Polish Acad. Sci., Warsaw, 2004.

[Ku] T.Z. Kuo, Characterizations of v-sufficiency of jets. Topology 11 (1972), 115-131.

[KOS] K. Kurdyka, P. Orro, S. Simon, Semialgebraic Sard theorem for generalized critical values, J. Differential Geometry 56 (2000), 67-92.

[Ma] J.N. Mather, Notes on topological stability, Harvard University 1970.

[Mi] J. Milnor, Singular points of complex hypersurfaces, Ann. of Math. Studies 61, Princeton 1968.

[Ne] A. NÉmethi, Lefschetz theory for complex affine varieties. Rev. Roumaine Math. Pures Appl. 33 (1988), no. 3, 233-250.

[NZ] A. NÉmethi, A. Zaharia, On the bifurcation set of a polynomial and Newton boundary, Publ. RIMS 26 (1990), 681-689.

[Pa1] A. PARUsińsKI, On the bifurcation set of complex polynomial with isolated singularities at infinity, Compositio Math. 97 (1995), no. 3, 369-384.

[Pa2] A. PARUsińsKI, A note on singularities at infinity of complex polynomials, Simplectic singularities and geometry of gauge fields, Banach Center Publ. 39 (1997), 131-141.

[PZ] A. L. PĂUNESCU, A. ZAHARIA, On the Eojasiewicz exponent at infinity for polynomial functions, Kodai Math. J. 20 (1997), no. 3, 269-274.

[Ph] F. Pham, Vanishing homologies and the $n$ variable saddlepoint method, Arcata Proc. of Symp. in Pure Math. vol. 40, II (1983), 319-333.

[Ra] P.J. RABIER, Ehresmann fibrations and Palais-Smale conditions for morphisms of Finsler manifolds. Ann. of Math. (2) 146 (1997), no. 3, 647-691.

[ST] D. Siersma, M. TibăR, Singularities at infinity and their vanishing cycles, Duke Math. Journal 80 (3) (1995), 771-783.

[Ti1] M. TIBĂR, On the monodromy fibration of polynomial functions with singularities at infinity. C. R. Acad. Sci. Paris Sér. I Math. 324 (1997), no. 9, 1031-1035.

[Ti2] M. TIBĂR, Topology at infinity of polynomial mappings and Thom regularity condition. Compositio Math. 111 (1998), no. 1, 89-109.

[Ti3] M. TiBăR, Regularity at infinity of real and complex polynomial maps, Singularity Theory, the C.T.C. Wall Anniversary Volume, LMS Lecture Notes Series 263 (1999), 249-264. Cambridge University Press.

[Ti4] M. TiB̆̆R, Asymptotic equisingularity and topology of complex hypersurfaces. Internat. Math. Res. Notices 1998, no. 18, 979-990.

[Ti5] M. TiBăR, Polynomials and Vanishing Cycles, Cambridge Tracts in Mathematics 170, Cambridge University Press 2007.

[TY] M. TibăR, Chen YIng, Bifurcation values of mixed polynomials, August 2010, preprint arXiv:1011.4884v1.

[TZ] M. Tibăr, A. ZAharia, Asymptotic behaviour of families of real curves, Manuscripta Math. 99 (1999), no. 3, 383-393.

[Wh] H. Whitney, Local properties of analytic varieties. 1965 Differential and Combinatorial Topology (A Symposium in Honor of Marston Morse) pp. 205-244 Princeton Univ. Press, Princeton, N. J. 
icmC, Universidade de São Paulo, Av. Trabalhador São-Carlense, 400 - CP Box 668, 13560-970 São Carlos, São Paulo, Brazil and laboratoire Painlevé, UMr 8524 CNRS, Université de Lille 1, 59655 Villeneuve D'AscQ, France.

E-mail address: lrgdias@icmc.usp.br

icmC, Universidade de São Paulo, Av. Trabalhador São-Carlense, 400 - CP Box 668, 13560-970 São Carlos, São PaUlo, Brazil.

E-mail address: maasruas@icmc.usp.br

Mathématiques, Laboratoire Painlevé, UMR 8524 CNRS, Université Lille 1, 59655 Villeneuve D'AscQ, France.

E-mail address: tibar@math.univ-lille1.fr 